\title{
TYPE RADICALS
}

\author{
by W. G. LEAVITT
}

(Received 16 December, 1966)

1. The lower radical of a module type. For a ring $R$ with unit, the module type $t(R)$ was defined in [6] as follows: $t(0)=0 ; t(R)=d$ if every free $R$-module has invariant rank; $t(R)=(c, k)$ for integers $c, k \geqq 1$ if every free $R$-module of rank $<c$ has invariant rank, while a free module of rank $h \geqq c$ has rank $h+n k$ for any integer $n \geqq 0$. The module types form a lattice under the ordering $0<(c, k)<d$ and $\left(c^{\prime}, k^{\prime}\right) \leqq(c, k)$ if and only if $c^{\prime} \leqq c$ and $k^{\prime} \mid k$. Two of the basic theorems on types are:

A. [6; Theorem 2, p. 115] If $R \rightarrow R^{\prime}$ is a unit-preserving homomorphism, then $t\left(R^{\prime}\right) \leqq t(R)$.

B. [6; Theorem 3, p. 116]

$$
t\left(\sum_{1}^{n} \oplus R_{i}\right)=\bigcup_{1}^{n} t\left(R_{i}\right) .
$$

We recall that in the definition of the Kurosh lower radical (as modified by Sulinski, Anderson and Divinsky in [10]) for a class $\mathscr{H}$ of rings, the class $\mathscr{H}_{1}$ is the homomorphic closure of $\mathscr{M}$, and for any ordinal $\beta>1$ the class $\mathscr{M}_{\beta}$ consists of rings $R$ such that every nonzero homomorphic image has a non-zero ideal in $\mathscr{M}_{\alpha}$ for some $\alpha<\beta$. Then the lower radical class defined by $\mathscr{M}$ is $\mathscr{L}(\mathscr{M})=\cup \mathscr{A}_{\beta}$, taken over all ordinals $\beta$. We note that, in fact [10; Theorem 1, p. 420], $\mathscr{L}(\mathscr{M})=\mathscr{M}_{\omega}$, where $\omega$ is the smallest infinite ordinal. If we define $\mathscr{T}_{\alpha}=\{R \mid t(R) \leqq \alpha\}$, then, by Theorem A, $\left(\mathscr{T}_{\alpha}\right)_{1}=\mathscr{T}_{\alpha}$. We further note that $\mathscr{T}_{0}=\{0\}$ is already (trivially) a radical class. We shall require the following lemma.

Lemma 1. If an ideal $I$ of a ring $R$ has a unit, then $R=I \oplus I^{*}$, where $I^{*}$ is the annihilator of $I$ in $R$.

Proof. The Pierce decomposition relative to the unit of $I$ clearly yields $R=I+I^{*}$, and since $I$ has a unit this sum must be direct.

For use in the following theorem, and at several other points, we record the following:

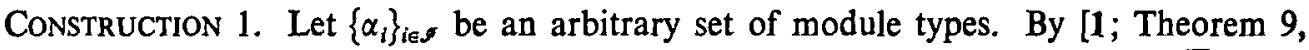
p. 130] there exists for each $i$ a ring $R_{i}$ with $t\left(R_{i}\right)=\alpha_{i}$. Let $R$ be the direct sum $\sum_{i \in \zeta} \oplus R_{i}$. Note that if $\mathscr{I}$ is infinite then $R$ does not have module type (since it has no unit), but nevertheless every non-zero image has a non-zero ideal with type. This follows from the fact that if $I$ is a proper ideal of $R$ then at least one $R_{i} \ddagger I$. Thus $R / I$ has a non-zero ideal $R+I / I$ $\cong R_{i} / R_{i} \cap I$, and, by Theorem $A, t\left(R_{i} / R_{i} \cap I\right) \leqq \alpha_{i}$.

THEOREM 1. $\mathscr{L}\left(\mathscr{T}_{\alpha}\right)=\left(\mathscr{T}_{\alpha}\right)_{2}$, and for $\alpha>0, \mathscr{L}\left(\mathscr{T}_{\alpha}\right) \neq\left(\mathscr{T}_{\alpha}\right)_{1}$. 
Proof. For the first statement it is sufficient to show that $\left(\mathscr{T}_{\alpha}\right)_{3}=\left(\mathscr{T}_{\alpha}\right)_{2}$. Thus suppose there exists $R \in\left(\mathscr{T}_{\alpha}\right)_{3}, R \notin\left(\mathscr{T}_{\alpha}\right)_{2}$. Then, by definition, $R$ has an image $\bar{R}$ with a non-zero ideal $l \in\left(\mathscr{T}_{\alpha}\right)_{2}$ but no ideal in $\left(\mathscr{T}_{\alpha}\right)_{1}$. Since $I \in\left(\mathscr{T}_{\alpha}\right)_{2}$ it has a non-zero ideal $J \in\left(\mathscr{T}_{\alpha}\right)_{1}=\mathscr{T}_{\alpha}$. Thus, by Lemma $1, I=J \oplus J^{*}$. Let $x \in J$ and $y \in \bar{R}$; then $x y \in I$ so that $x y=z+z^{*}$ for some $z \in J$ and $z^{*} \in J^{*}$. But if $e$ is the unit of $J$, this yields $x y=e x y=e z=z \in J$. Similarly $y x \in J$, so $J$ is an ideal of $\bar{R}$, contradicting the condition that $\bar{R}$ should have no ideals from $\mathscr{T}_{\alpha}$.

To establish the second statement of the theorem, let $R$ be the ring of Construction 1 with $\mathscr{I}$ infinite and all $\alpha_{i}=\alpha$. Then $R \notin \mathscr{T}_{\alpha}$ but every non-zero image has a non-zero ideal $I$ with $t(I) \leqq \alpha$. Thus $R \in\left(\mathscr{T}_{\alpha}\right)_{2}$.

2. The lower maxit radicals. In [9] the module type was used to construct for a general ring an invariant (called the " maxit" of the ring) which coincides with the module type for rings with unit. In the present paper we shall sharpen the definition of [9] in the following way: we shall extend the module type lattice to a lattice of maxits by permitting $c$ and $k$ in $(c, k)$ to take on values $\omega$ and 0 respectively, in addition to all positive integers. The order in this extended lattice (and hence the lattice operations) is defined as for module types, noting that $c \leqq \omega$ for all $c$ and $k \mid 0$ for all $k$. We now define the maxit $m(R)$ of a ring $R$ as follows:

(i) Whenever $R$ has module type, $m(R)=t(R)$.

(ii) For all other rings, let $\mathscr{W}$ be the set consisting of all modular ideals of $R$ together with $R$ itself; then $m(R)=\bigcup_{I \in W} m(R / I)$.

REMARK 1. From this definition it follows that a ring $R$ is a Brown-McCoy radical ring if and only if $m(R)=0$. This is clear since $R$ is a Brown-McCoy radical if and only if it has no modular ideals [8, p. 134]. But then $m(R)=m(R / R)=t(0)=0$.

REMARK 2. On the other hand, since in the extended lattice such maxits as $(\omega, k)$ are now available, it is clear that $m(R)=d$ if and only if there exists some $I \in \mathscr{W}$ such that $t(R / I)=d$.

REMARK 3. From the definition of the maxit it is also clear that, if $\bar{R}$ is a homomorphic image of $R$, then $m(\bar{R}) \leqq m(R)$.

REMARK 4. It is easily seen that the method of proof of [9; Theorem 3.2, p. 131] can be applied to yield $m\left(\sum_{1}^{n} \oplus R_{i}\right)=\bigcup_{1}^{n} m\left(R_{i}\right)$. This result will be extended (see Theorem 2) to infinite direct sums or complete direct sums (to be written $\sum_{c} \oplus R_{i}$ ).

For a given ring $R$, define

$$
G(a)=\left\{x a-x+a y-y+\sum\left(x_{i} a y_{i}-x_{i} y_{i}\right)\right\} \text { for all } x, y, x_{i}, y_{i} \in R .
$$

LEMMA 2. $m(R)=\bigcup_{a \in R} t(R / G(a))$.

Proof. If $I$ is a modular ideal of $R$, then there exists an identity $a$ of $R$ modulo $I$. Thus $G(a) \subseteq I$ and from the natural homomorphism $R / G(a) \rightarrow R / I$ and Theorem $\mathrm{A}, t(R / I)$ $\leqq t(R / G(a))$. Thus $m(R)=\bigcup_{I \in \mathscr{W}} t(R / I) \leqq \bigcup_{a \in R} t(R / G(a))$. 
But also $G(a) \in \mathscr{W}$; so we have the reverse inequality.

Let $\left\{a_{i}\right\}(i=1,2, \ldots, n)$ be a set of members of $R$, and write

$$
b=\sum_{k=1}^{n}(-1)^{k+1} \sum_{i_{1}<\ldots<i_{k}} a_{i_{1}} \ldots a_{i_{k}} .
$$

LEMMA 3. $G(b) \subseteq \bigcap_{1}^{n} G\left(a_{i}\right)$.

Proof. For a given $i$, let $f$ be any polynomial in $\left\{a_{i}\right\}$ over the integers not containing $a_{i}$ as a term. It is clear that $f\left(a_{1} \ldots a_{i} \ldots a_{n}\right)-f\left(a_{1} \ldots 1 \ldots a_{n}\right) \in G\left(a_{i}\right)$, where " 1 " simply indicates the deletion of any $a_{i}$ from any term. But it is easy to see from (2) that if 1 is substituted for $a_{i}$ in $b-a_{i}$ the result is zero. Hence $b-a_{i} \in G\left(a_{i}\right)$. Thus $b$ is a unit modulo $G\left(a_{i}\right)$ for each $i$, and so is a unit modulo $\cap G\left(a_{i}\right)$.

Proposition 1. (a) If $m(R)=(c, k)$ for integers $c, k \geqq 1$, then there exists an ideal $I$ of $R$ such that $t(R / I)=(c, k)$.

(b) If $m(R)=(\omega, k)$, then for some infinite ascending chain of integers $c_{1}<c_{2}<\ldots$ there exists a chain $I_{1} \supset I_{2} \supset \ldots$ of ideals such that $t\left(R / I_{i}\right)=\left(c_{i}, k\right)$.

(c) Similarly, if $m(R)=(c, 0)$, there is an infinite ascending chain $\left\{k_{i}\right\}$ of proper divisors and $t\left(R / I_{i}\right)=\left(c, k_{i}\right)$.

(d) Similarly, if $m(R)=(\omega, 0)$, there are ascending chains $\left\{c_{i}\right\}$ and $\left\{k_{i}\right\}$ and $t\left(R / I_{i}\right)=\left(c_{i}, k_{i}\right)$.

Proof. (a) Let $m(R)=(c, k)$ for $c, k \geqq 1$. Choose one $a_{i}$ (if such exists) for which $t\left(R / G\left(a_{i}\right)\right)=\alpha_{i}$ for each $\alpha_{i} \leqq(c, k)$. The set $\left\{a_{i}\right\}$ so chosen is finite, say $i=1, \ldots, n$. Define $b$ by (2); then it follows from Lemma 3 and Theorem A that $t(R / G(b)) \geqq \bigcup_{1}^{n} t\left(R / G\left(a_{i}\right)\right)$. But, by Lemma 2, the right side of this inequality is $m(R)$, and since by definition $t(R / G(b))$ $\leqq m(R)$, equality follows.

(b) If $m(R)=(\omega, k)$, then we again have only a finite set $k_{i} \mid k$ for which a choice $a_{i}$ exists with $t\left(R / G\left(a_{i}\right)\right)=\left(c_{i}, k_{i}\right)$ for some $c_{i}$. Now $k$ is clearly the least common multiple of the $\left\{k_{i}\right\}$. Thus if $b_{1}$ is the $b$ defined by (2) for this set of $\left\{a_{i}\right\}$, then, by the same argument as in $(a)$ above, $t\left(R / G\left(b_{1}\right)\right) \geqq(c, k)$ for some $c$. But $t\left(R / G\left(b_{1}\right)\right)<(\omega, k)$, so we must have $t\left(R / G\left(b_{1}\right)\right)=\left(c_{1}, k\right)$ for some $c_{1}$. Since $m(R)=(\omega, k)$, there must, by Lemma 2, exist some $b^{\prime}$ such that $t\left(R / G\left(b^{\prime}\right)\right)=\left(c^{\prime}, k^{\prime}\right)$ for which $c^{\prime}>c_{1}$ (of course with some $k^{\prime} \mid k$ ). Defining, as in (2), $b_{2}=b_{1}+b^{\prime}-b_{1} b^{\prime}$, we have $G\left(b_{2}\right) \subseteq G\left(b_{1}\right) \cap G\left(b^{\prime}\right)$ so that $t\left(R / G\left(b_{2}\right)\right) \geqq\left(c_{1}, k\right) \cup\left(c^{\prime}, k^{\prime}\right)$ $=\left(c^{\prime}, k\right)$. Thus $t\left(R / G\left(b_{2}\right)\right)=\left(c_{2}, k\right)$ for some $c_{2}>c_{1}$. The process clearly continues. It is also clear that similar constructions exist for cases $(c)$ and $(d)$.

Let $I$ be an ideal of $R$ and $a \in I$. Write $G_{I}(a)$ for the ideal of $I$ defined by (1), restricting $x, y, x_{i}, y_{i}$ to be elements of $I$.

LEMMA 4. $G_{I}(a)=G(a) \cap I$.

Proof. Clearly $G_{I}(a) \subseteq G(a) \cap I$; so suppose that $z \in G(a) \cap I$. Then $z-a z a=(z-z a)$ $+(z a-a z a) \in G_{I}(a)$. But from $z \in G(a)$ and $a \in I$ it follows that $a z a \in G_{I}(a)$. Thus $z \in G_{I}(a)$, and so $G(a) \cap I \subseteq G_{l}(a)$. 
Let $\left\{R_{j}\right\}_{j \in J}$ be a set of rings. If either $R=\sum \oplus R_{j}$ or $R=\sum_{c} \oplus R_{j}$, then from the natural homomorphism $R \rightarrow R_{j}$ it follows, by Remark 3, that $\mathrm{U} m\left(R_{j}\right) \leqq m(R)$. Let $a_{j} \in R_{j}$ be the projection of $a \in R$, and write $G_{j}=G_{R}\left(a_{j}\right)$.

THEOREM 2. (a) If $R=\sum \oplus R_{j}$, then $m(R)=\bigcup_{j \in J} m\left(R_{j}\right)$.

(b) If $R=\sum_{c} \oplus R_{j}$ and there exists $a \in R$ such that $\bigcup_{t}\left(R_{j} / G_{j}\right)=(\omega, k),(c, 0)$, or $(\omega, 0)$, then $m(R)=d$. Otherwise $m(R)=\bigcup_{j \in J} m\left(R_{j}\right)$.

Proof. (a) Let $a \in \sum \oplus R_{j}$; then $a=\sum_{1}^{n} a_{j}$. Thus $G(a)=\left(\sum_{1}^{n} \oplus G_{j}\right) \oplus\left(\sum_{j>n} \oplus R_{j}\right)$ and it is easily seen that $R / G(a) \cong \sum_{1}^{n} \oplus R_{j} / G_{j}$. From Theorem $\mathrm{B}$, it follows that $t(R / G(a))$ $=\bigcup_{1}^{n} t\left(R_{j} / G_{j}\right) \leqq \bigcup_{j \in J} m\left(R_{j}\right)$. Since $a$ was arbitrary, by Lemma 2, we have $m(R) \leqq \bigcup_{j \in J} m\left(R_{j}\right)$ and hence equality.

(b) Let $a \in \sum_{c} \oplus R_{j}$; then again it is easy to see that $G(a)=\sum_{c} \oplus G_{j}$ and $R / G(a) \cong \sum_{c} \oplus R_{j} / G_{j}$. From the projection $R / G(a) \rightarrow R_{j} / G_{j}$ we have $t\left(R_{j} / G_{j}\right) \leqq t(R / G(a))$. Thus, if $\bigcup_{t}\left(R_{j} / G_{j}\right)$ is not a module type, then $t(R / G(a))=d$ and so $m(R)=d$.

Now if $t\left(R_{j} / G_{j}\right)=0$ for all $j \in J$, then all $R_{j}=G_{j}$ and so $R=G(a)$. If this is true for all $a \in R$, then, by Lemma $2,0=m(R)=\bigcup \begin{aligned} & \\ & m\left(R_{j}\right)\end{aligned}$.

Thus suppose for a given $a \in R$ we have $\bigcup t\left(R_{j} / G_{j}\right)=(c, k)$, so that all $t\left(R_{j} / G_{j}\right) \leqq(c, k)$. Then if $G_{j} \neq R_{j}$, by the matrix criterion for rings of given module type (see [1] or [6]), there exist $c+k$ by $c$ and $c$ by $c+k$ matrices $A_{j}, B_{j}$ with elements in $R_{j}$ such that

$$
A_{j} B_{j}-a_{j} I_{c+k} \equiv 0\left(\bmod G_{j}\right) \text { and } B_{j} A_{j}-a_{j} I_{c} \equiv 0\left(\bmod G_{j}\right) \text {, }
$$

where we write $a_{j} I_{c}$ for the diagonal matrix with $a_{j}$ on the diagonal (that is, a unit matrix over $R_{j}$ modulo $G_{j}$ ).

Let $A=\sum_{c} \oplus A_{j}$ and $B=\sum_{c} \oplus B_{j}$ (note: insert a zero matrix whenever $G_{j}=R_{j}$ ). Then clearly $A B-a I_{c+k}$ and $B A-a I_{c} \equiv 0(\bmod G(a))$; so, by the matrix criterion for module type, $t(R / G(a)) \leqq(c, k)$. Thus $m(R)=\bigcup_{a} t(R / G(a)) \leqq \bigcup_{a} \bigcup_{j \in J} t\left(R_{j} / G_{j}\right)$. By reversing the order of unions in the last expression, this yields $m(R) \leqq \bigcup \begin{aligned} & \\ & \text { (n) }\end{aligned}\left(R_{j}\right)$ and hence equality.

We recall that a class of rings is called hereditary if it includes all ideals of all of its members.

THEOREM 3. The class $\mathscr{M}_{\alpha}=\{R \mid m(R) \leqq \alpha\}$ is a hereditary class.

Proof. Let $R \in \mathscr{M}_{a}$ and $a \in I$, where $I$ is an ideal of $R$. Since $m(R) \leqq \alpha$, it follows that $t(R / G(a)) \leqq \alpha$. The Pierce decomposition $x=a x-(a x-x)$ shows that $R=I+G(a)$. Thus $R / G(a) \cong I / I \cap G(a)$ and by Lemma 4 this equals $I / G_{I}(a)$. From Lemma 2 it follows that $m(I) \leqq \alpha$.

CoROLlary 1. If $m(R)<d$, then, for an ideal I of $R$, we have the following results.

(a) If $I$ is Noetherian, it is included in the Brown-McCoy radical of $R$. 
(b) If $I$ is commutative, it is included in the Jacobson radical of $R$.

(c) If $I$ is Artinian, it is included in the nil radical of $R$.

Proof. Note first that, if $I$ satisfies one of the conditions of $(a),(b)$ or $(c)$, then so does every homomorphic image of $I$. Now, by Theorem $3, m(I)<d$ and, if $m(I) \neq 0$, then $I$ has a non-zero homomorphic image $I$ with $t(\bar{I}) \leqq m(I)$. But by [4; p. 32] if $I$ is Noetherian, and hence by [4; Theorem 29, p. 71] if $I$ is Artinian, or by $[2 ;$ p. 563] if $I$ is commutative, then $t(I)=d$, contradicting $t(\bar{I}) \leqq m(I)$. Thus $m(I)=0$ and so $I$ is a Brown-McCoy radical ring. Hence if $I$ is commutative it is a Jacobson radical ring, or if it is Artinian it is nil.

THEOREM 4. $\mathscr{L}\left(\mathscr{M}_{\alpha}\right)=\left(\mathscr{M}_{\alpha}\right)_{2}$ and is a hereditary radical class.

Proof. By Remark 3, $\mathscr{M}_{\alpha}=\left(\mathscr{M}_{\alpha}\right)_{1}$ and, by Theorem 3, it is a hereditary class. Also any nilpotent ring $R$ is Brown-McCoy radical, so $m(R)=0 \leqq \alpha$. Thus it follows from [10; Theorem 2, p. 420] that $\mathscr{L}\left(\mathscr{M}_{\alpha}\right)=\left(\mathscr{M}_{\alpha}\right)_{2}$. Now, by [3; Theorem 1.4, p. 29], the lower radical of any hereditary class is hereditary, and so $\mathscr{L}\left(\mathscr{M}_{\alpha}\right)$ is a hereditary class.

Although $\mathscr{M}_{\alpha}$ may not in general be a radical class, the ideals $I$ of $R$ satisfying $I \in \mathscr{M}_{\alpha}$ or $R / I \in \mathscr{M}_{\alpha}$ have certain maximal properties. We conclude this section by recording these properties.

PROPOSITION 2. (a) Every ring $R$ has an ideal I maximal relative to $I \in \mathscr{M}_{a}$.

(b) If $R$ has an ideal $I$ with $m(I)=\alpha$, then it has an ideal maximal relative to this property.

Proof. To show that Zorn's Lemma applies, let $\left\{I_{i}\right\}$ be a chain of ideals with $m(I) \leqq \alpha$ (or $=\alpha$ in case $(b)$ ). Let $I=\bigcup I_{i}$, so that, by Theorem 3, $m(I) \geqq \bigcup m\left(I_{i}\right)$. Suppose $m(I) \pm \alpha$ (or $\neq \alpha$ in case $(b)$ ). Then there exists some $a \in I$ such that $t\left(I / G_{I}(a)\right)=\beta \pm \alpha$ (or $>\alpha$ in case $(b)$ ). Now $a \in I$ and so $a \in I_{i}$ for some $I_{i}$ in the chain. Writing $G_{i}=G_{I_{i}}(a)$, we obtain from Lemma $4 G_{i}=G_{I}(a) \cap I_{i}$, and, as in the proof of Theorem $3, I / G_{I}(a) \cong I_{i} / G_{i}$. Thus $t\left(I_{i} / G_{i}\right)=\beta$, violating the definition of $I_{i}$. Hence $m(I) \leqq \alpha($ or $=\alpha$ in case $(b))$ and so Zorn's Lemma applies.

Proposition 3. If $R$ contains an ideal $H$ such that $t(R / H)=\alpha$, then it contains an ideal maximal relative to this property.

Proof. If $\alpha=0$, then $H=R$ satisfies the proposition. Thus suppose $\alpha>0$. To show that Zorn's Lemma applies, let $H=\bigcup H_{i}$ for a chain $\left\{H_{i}\right\}$ for which $t\left(R / H_{i}\right)=\alpha$. By Theorem $\mathrm{A}, t(R / H) \leqq t\left(R / H_{i}\right)=\alpha$, and suppose $t(R / H)=\beta<\alpha$. Thus $\beta \neq d$ and if $\beta=0$ then $H=R$. But for any $H_{i}$, if $a_{i}$ is the identity of $R$ modulo $H_{i}$, this means that $a_{i}$ belongs to some $H_{j}$ in the chain. Now $a_{i} \notin H_{i}$; so $H_{j} \ddagger H_{i}$ and hence $H_{i} \subseteq H_{j}$. But this is also impossible, for then $G\left(a_{i}\right) \subseteq H_{j}$ and thus, since $a_{i} \in H_{j}, R \subseteq H_{j}$.

We therefore suppose that $\beta=(c, k)$ and let $a$ be an identity of $R$ modulo $H$. As was remarked in the proof of Theorem 2, the matrix criterion then requires the existence of $c+k$ by $c$ and $c$ by $c+k$ matrices $S$ and $T$ such that

$$
U=S T-a I_{c+k} \equiv 0(\bmod H) \text { and } V=T S-a I_{c} \equiv 0(\bmod H) .
$$

Now there is only a finite number of elements in the matrices $U$ and $V$, and since all are 
contained in $H$, they must in fact all be contained in some $H_{i}$ of the chain. If $a_{i}$ is the identity of $R$ modulo $H_{i}$, then $a a_{i}-a \in H_{i}$. On the other hand, $a$ is an identity of $R$ modulo $H$, so that $a a_{i}-a_{i} \in H$ and so is in $H_{j}$, for some $H_{j}$ in the chain. One of the two must be larger, say $H_{j} \supseteq H_{i}$. Then $a$ is a unit of $R$ modulo $H_{j}$, and since $U, V \equiv 0\left(\bmod H_{j}\right)$, we have $t\left(R / H_{j}\right) \leqq \beta$, contradicting the definition of $H_{j}$. We conclude that $t(R / H)=\alpha$, and so Zorn's Lemma applies.

Corollary 2. For $\alpha$ a module type, if $m(R / H)=\alpha$ for some ideal $H$ of $R$, then $R$ contains an ideal maximal relative to this property.

Proof. If $\alpha=0$, let $H=\mathrm{U} H_{i}$ for a chain of ideals with $m\left(R / H_{i}\right)=0$. By Remark 3, $m(R / H) \leqq m\left(R / H_{l}\right)=0$. Thus $m(R / H)=0$, and so Zorn's Lemma applies. Thus suppose that $m(R / H)=\alpha>0$. By Proposition 1 (or Remark 2 if $\alpha=d$ ), there exists an ideal $I \supseteq H$ such that $t(R / I)=\alpha$. By Proposition 3, we may assume that $I$ is maximal relative to this property. Suppose there could exist $H_{1} \supset I$ such that $m\left(R / H_{1}\right)=\alpha$. By the same argument, there would then exist $I_{1} \supseteq H_{1}$ such that $t\left(R / I_{1}\right)=\alpha$, violating the maximality of $I$.

3. Simple type radicals. Let $\mathscr{W}$ be the set consisting of all maximal modular ideals of $R$, together with $R$ itself. Define $\bar{m}(R)=\bigcup_{I \in W} m(R / I)$, and write $\overline{\mathscr{M}}_{x}=\{R \mid \bar{m}(R) \leqq \alpha\}$. Then, by definition, $\mathscr{M}_{\alpha} \subseteq \overline{\mathscr{M}}_{\alpha}$.

LEMMA 5. $\bar{m}(R / I) \leqq \bar{m}(R)$ for all ideals $I$ of $R$.

Proof. If $H / I$ is maximal in $R / I$, then $H$ is maximal in $R$. Since $(R / I) /(H / I) \cong R / H$, it follows that $\bar{m}(R / I)=\bigcup_{t}(R / H)$, the union being taken over all $H \supseteq I$; hence result.

LEMMA 6. If $I$ is an ideal of $R$, then $\bar{m}(I) \leqq \bar{m}(R)$.

Proof. If $I$ has no maximal modular ideals, then $\bar{m}(I)=0$ and the Lemma is satisfied trivially. Now it is well-known that $H_{1}$ is a maximal modular ideal of $I$ if and only if $H_{1}=I \cap H$ for some maximal modular ideal $H$ of $R$. Thus $I \nsubseteq H$, so that $R=I+H$, and hence $I / H_{1} \cong R / H$. It follows that $\bar{m}(I)=\bigcup m\left(I / H_{1}\right) \leqq \bar{m}(R)$.

Let $\mathscr{S}$ be the class of all simple rings, partitioned into $\mathscr{S}_{\alpha}=\{R \in \mathscr{P} \mid m(R)=\bar{m}(R) \leqq \alpha\}$ and $\mathscr{S}_{\alpha}^{\prime}=\mathscr{S}_{-} \mathscr{S}_{\alpha}$. One way to use this partitioning would be to consider $\mathscr{L}\left(\mathscr{S}_{\alpha}\right)$. Since $\mathscr{S}_{\alpha} \subseteq \mathscr{M}_{\alpha}$ we have $\mathscr{L}\left(\mathscr{S}_{\alpha}\right) \subseteq \mathscr{L}\left(\mathscr{M}_{\alpha}\right)$. However, let $\mathscr{N}$ be the class of all zero rings (rings with trivial multiplication: $x y=0$ for all $x, y$ in the ring) without minimal ideals. Note that $\mathscr{N}$ is non-empty since we can impose a trivial multiplication on any additive abelian group without minimal subgroups (such as the additive group of the integers). Now it is known [3; Theorem 1.15, p. 39] that, if a class $\mathscr{U}$ is homomorphically closed and the class $\mathscr{V}$ is hereditary, then $\mathscr{U} \cap \mathscr{V}=0$ implies that $\mathscr{L}(\mathscr{U}) \cap \mathscr{V}=0$. Clearly the class $\mathscr{N}$ is hereditary and $\left(\mathscr{S}_{a}\right)_{1} \cap \mathscr{N}$ $=0$, so that $\mathscr{L}\left(\mathscr{S}_{\alpha}\right) \cap \mathcal{N}=0$. On the other hand the zero rings are Brown-McCoy radical; so $\mathscr{N} \subseteq \mathscr{H}_{\alpha}$. Thus $\mathscr{L}\left(\mathscr{S}_{\alpha}\right)$ is properly contained in $\mathscr{L}\left(\mathscr{M}_{\alpha}\right)$. We might ask the relation of $\mathscr{L}\left(\mathscr{T}_{\alpha}\right)$ to $\mathscr{L}\left(\mathscr{S}_{\alpha}\right)$. However, these classes are not comparable: if $R$ is a simple radical ring, then $R \in \mathscr{S}_{\alpha}$ but $R \notin \mathscr{L}\left(\mathscr{T}_{\alpha}\right)$; on the other hand, the ring of integers $Z \in \mathscr{T}_{\mathrm{d}}$ but has no minimal ideals and so is not in $\mathscr{L}\left(\mathscr{S}_{d}\right)$. 
The upper radical defined by this partition [5; p. 22] can, however, be used to characterize $\overline{\mathscr{M}}_{\alpha}$. Write $\mathscr{U}\left(\mathscr{S}_{\alpha}^{\prime}\right)$ for the upper radical class defined by $\mathscr{S}_{\alpha}^{\prime}$. (Recall $[5 ;$ p. 17] that $\mathscr{U}\left(\mathscr{S}_{\alpha}^{\prime}\right)=\left\{R \mid\right.$ every non-zero homomorphic image $\left.R / I \notin \mathscr{S}_{\alpha}^{\prime}\right\}$.)

THEOREM 5. $\overline{\mathscr{M}}_{\alpha}=\mathscr{U}\left(\mathscr{S}_{\alpha}^{\prime}\right)$ and is thus a hereditary radical class.

Proof. Let $R \in \overline{\mathscr{M}}_{\alpha}$, so that $\bar{m}(R)=\beta \leqq \alpha$. Then, by definition, for any simple image $R / H$ we have $m(R / H) \leqq \beta$, and so $R$ has no images in $\mathscr{S}_{\alpha}^{\prime}$. Thus $\overline{\mathscr{M}}_{\alpha} \subseteq \mathscr{U}\left(\mathscr{S}_{\alpha}^{\prime}\right)$. On the other hand, if $R \in \mathscr{U}\left(\mathscr{S}_{\alpha}^{\prime}\right)$ but $R \notin \overline{\mathscr{M}}_{\alpha}$, then we would have $\bar{m}(R)=\beta \pm \alpha$. There would then exist some maximal modular $H$ such that $\bar{m}(R / H)=t(R / H) \$ \alpha$. Thus $R / H \in \mathscr{S}_{a}^{\prime}$, contradicting the definition of $\mathscr{U}\left(\mathscr{S}_{\alpha}^{\prime}\right)$. Hence $\overline{\mathscr{M}}_{\alpha}=\mathscr{U}\left(\mathscr{S}_{\alpha}^{\prime}\right)$ and so is a radical class, and by Lemma 6 it is hereditary.

We remark that, since $\mathscr{T}_{\alpha} \subseteq \mathscr{M}_{\alpha} \subseteq \overline{\mathscr{M}}_{\alpha}$ for all module types $\alpha$, it follows that $\mathscr{L}\left(\mathscr{T}_{\alpha}\right)$ $\subseteq \mathscr{L}\left(\mathscr{M}_{\alpha}\right) \subseteq \overline{\mathscr{M}}_{\alpha}$. The first inequality is always proper since every $\mathscr{M}_{\alpha}$ contains all BrownMcCoy radical rings. However, it is an open question whether or not $\overline{\mathscr{M}}_{\alpha}$ differs from $\mathscr{L}\left(\mathscr{M}_{\alpha}\right)$ (or even from $\mathscr{M}_{\alpha}$ ). Of course, trivially, $\mathscr{M}_{d}=\mathscr{L}\left(\mathscr{M}_{d}\right)=\overline{\mathscr{M}}_{\mathrm{d}}$ since $\mathscr{M}_{\mathrm{d}}$ is the class of all rings.

We may also ask whether or not these radical classes differ for different $\alpha$. There are a number of open questions in this regard, but we may state the following:

1. If $\alpha$ is a module type, then $\mathscr{L}\left(\mathscr{T}_{\alpha}\right)$ is non-zero, and if $\alpha \neq \beta$, then $\mathscr{L}\left(\mathscr{T}_{\alpha}\right) \neq \mathscr{L}\left(\mathscr{T}_{\beta}\right)$.

Proof. We may assume that $\alpha \neq \beta$. If $\alpha=d$, then $\beta \neq d$ and any field is in $\mathscr{L}\left(\mathscr{T}_{d}\right)$ but not in $\mathscr{L}\left(\mathscr{T}_{\beta}\right)$. If $\alpha=(c, k)$, use the ring $V=V_{c, c+k}$ [1; Section 5, p. 221] (see also [6; Footnote 6, p. 130]) universal for rings of type $\alpha$; then $V \in \mathscr{T}_{\alpha}$. Suppose that $V \in \mathscr{L}\left(\mathscr{T}_{\beta}\right)$; then, by Theorem 1 , there exists a non-zero ideal $I$ of $V$ such that $t(I) \leqq \beta$. But, aside from its unit, $V$ has no elements which are even local identities. Thus $V$ itself is the only ideal of $V$ which has type, and $t(V)$ 主 $\beta$.

2. Since $\mathscr{M}_{0}$ is the class of all Brown-McCoy radical rings, $\mathscr{L}\left(\mathscr{M}_{0}\right)=\mathscr{M}_{0}$. Then, since $\mathscr{M}_{\beta}$, for any $\beta \neq 0$, contains rings with unit, $\mathscr{L}\left(\mathscr{M}_{0}\right) \neq \mathscr{L}\left(\mathscr{M}_{\beta}\right), \beta \neq 0$.

On the other hand, $\mathscr{L}\left(\mathscr{M}_{\beta}\right)$ for $\beta \neq d$ contains no fields, and, since $\mathscr{M}_{d}=\mathscr{L}\left(\mathscr{M}_{d}\right)$ is the class of all rings, $\mathscr{L}\left(\mathscr{M}_{\mathrm{d}}\right) \neq \mathscr{L}\left(\mathscr{M}_{\beta}\right), \beta \neq d$.

3. For given $\alpha \neq \beta$, if $\overline{\mathscr{M}}_{\alpha} \neq \overline{\mathscr{M}}_{\beta}$, then $\mathscr{L}\left(\mathscr{M}_{\alpha}\right) \neq \mathscr{L}\left(\mathscr{M}_{\beta}\right)$.

Proof. We can assume that $\overline{\mathscr{M}}_{\alpha} \neq \overline{\mathscr{M}}_{\beta}$. Thus there must exist some $R$ with $\bar{m}(R) \leqq \alpha$ and $\bar{m}(R) \leq \beta$. It follows that $R$ has a simple image $\bar{R}$ with $t(\bar{R}) \leqq \alpha$ and $t(\bar{R}) \leqq \beta$. Hence $\bar{R} \in \mathscr{M}_{\alpha}$, and, since it is simple, $\bar{R} \notin \mathscr{L}\left(\mathscr{M}_{\beta}\right)$.

4. When $\alpha=(c, k)$ and $\beta=\left(c^{\prime}, k^{\prime}\right)$, with $k \neq k^{\prime}$ (integers $\geqq 0$ ) and $c, c^{\prime}$ arbitrary (integers $\geqq 1$, or one or both equal to $\omega)$, then $\ddot{\mathscr{M}}_{\alpha} \neq \overline{\mathscr{M}}_{\beta}$ and so $\mathscr{L}\left(\mathscr{M}_{\alpha}\right) \neq \mathscr{L}\left(\mathscr{M}_{\beta}\right)$.

Proof. We may assume that $k \nmid k^{\prime}$ (so $k^{\prime} \neq 0$ ). If $k \neq 0$, there exists a simple ring $R$ [7; Theorem 2, p. 307] such that $t(R)=(1, k) \leqq \alpha, \$ \beta$. Thus $R \in \overline{\mathscr{M}}_{\alpha}, \notin \overline{\mathscr{M}}_{\beta}$. If $k=0$, we may use Construction 1 with $R=\sum_{1}^{\infty} \oplus R_{i}$, where the $R_{i}$ are simple with $t\left(R_{i}\right)=(1, i)$. Then $\bar{m}(R)=(1,0)$ and so again $R \in \overline{\mathscr{M}}_{\alpha}, \notin \overline{\mathscr{M}}_{\beta}$. 
5. The only remaining case is $\alpha=(c, k), \beta=\left(c^{\prime}, k\right)$, with $c \neq c^{\prime}$, say $c^{\prime}<c$. It is clear that if a simple ring exists for which $t(R)=(c, 1)$, then an argument similar to those of the preceding paragraph would show that $\overline{\mathscr{M}}_{\alpha} \neq \overline{\mathscr{M}}_{\beta}$. However, the existence of such rings for $c>1$ is an open question. A further question, in this case, is the following: even if $\overline{\mathscr{M}}_{\alpha}=\overline{\mathscr{M}}_{\beta}$, does it follow that $\mathscr{L}\left(\mathscr{M}_{\alpha}\right)=\mathscr{L}\left(\mathscr{A}_{\beta}\right)$ ?

\section{REFERENCES}

1. P. M. Cohn, Some remarks on the invariant basis property, Topology 5 (1966), 215-228.

2. J. Dieudonné, Sur le nombre de dimensions d'un module, C. R. Acad. Sci. Paris 215 (1942), 563-565.

3. A. E. Hoffman, The constructions of the general theory of radicals, Thesis, University of Nebraska (1966).

4. N. Jacobson, The theory of rings, Amer. Math. Soc. Mathematical Surveys, No. 2 (Providence, R.I., 1943).

5. A. G. Kurosh, Radicals in rings and algebras, Mat. Sb. N.S. 33 (1953), 13-26.

6. W. G. Leavitt, The module type of a ring, Trans. Amer. Math. Soc. 103 (1962), 113-130.

7. W. G. Leavitt, The module type of homomorphic images, Duke Math.J. 32 (1965), 305-312.

8. N. H. McCoy, Theory of rings (New York, 1964).

9. R. E. Peinado and W. G. Leavitt, The maxit and minit of a ring, Proc. Glasgow Math. Assoc. 7 (1966), 128-135.

10. A. Sulinski, R. Anderson, and N. Divinsky, Lower radical properties for associative and alternative rings, J. London Math. Soc. 41 (1966), 417-424.

UNIVERSITY OF NEBRASKA 